\title{
Minimally invasive surgical aortic valve replacement: The RALT approach
}

\section{Journal Article}

\section{Author(s):}

Van Praet, Karel M.; van Kampen, Antonia; Kofler, Markus; Richter, Gregor; Sündermann, Simon H.; Meyer, Alexander; Unbehaun, Axel; Kurz, Stephan; Jacobs, Stephan; Falk, Volkmar (D); Kempfert, Jörg

Publication date:

2020-09

Permanent link:

https://doi.org/10.3929/ethz-b-000426879

Rights / license:

Creative Commons Attribution-NonCommercial-NoDerivatives 4.0 International

Originally published in:

Journal of Cardiac Surgery 35(9), https://doi.org/10.1111/jocs.14756 


\title{
Minimally invasive surgical aortic valve replacement: The RALT approach
}

\author{
Karel M. Van Praet MD ${ }^{1,2}$ | Antonia van Kampen MD $^{1,2}$ | Markus Kofler MD ${ }^{1}$ | \\ Gregor Richter $\mathrm{MD}^{1}$ | Simon H. Sündermann $\mathrm{MD}, \mathrm{PhD}^{2,3}$ | \\ Alexander Meyer MD, PhD ${ }^{1,2,4}$ | Axel Unbehaun MD, $\mathrm{PhD}^{1,2}$ | Stephan Kurz $\mathrm{MD}^{1,3}$ | \\ Stephan Jacobs MD, PhD ${ }^{1,2} \mid$ Volkmar Falk MD, PhD ${ }^{1,2,3,4,5}$ | Jörg Kempfert MD, PhD ${ }^{1,2}$
}

\author{
${ }^{1}$ Department of Cardiothoracic and Vascular \\ Surgery, German Heart Center Berlin, Berlin, \\ Germany \\ ${ }^{2}$ German Center for Cardiovascular Research \\ (DZHK), Partner Site Berlin, Berlin, Germany \\ ${ }^{3}$ Department of Cardiothoracic Surgery, \\ Charité-Universitätsmedizin Berlin, Berlin, \\ Germany \\ ${ }^{4}$ Institute of Health (BIH), Berlin, Germany \\ ${ }^{5}$ Department of Health Sciences, Translational \\ Cardiovascular Technologies, ETH Zürich, \\ Zürich, Switzerland \\ Correspondence \\ Karel M. van Praet, MD, Department of \\ Cardiothoracic and Vascular Surgery, German \\ Heart Center Berlin (Deutsches Herzzentrum \\ Berlin), Augustenburger Platz 1, D-13353 \\ Berlin, Germany. \\ Email: vanpraet@dhzb.de
}

\begin{abstract}
Less-invasive techniques for cardiothoracic surgical procedures are designed to limit surgical trauma, but the technical requirements and preoperative planning are more demanding than those for conventional sternotomy. Patient selection, interdisciplinary collaboration, and surgical skills are key factors for procedural success. Aortic valve replacement is frequently performed through an upper hemisternotomy, but the right anterior minithoracotomy represents an even less traumatic, technical advancement. Preoperative assessment of the ascending aorta in relation to the sternum is mandatory to select patients and the intercostal access site. This description of the surgical technique focuses on the specific procedural details including the obligatory planning with computed tomography and our cannulation strategy. We also sought to define the anatomical ascending aorta-sternal relationship, as it is of utmost importance in preoperative computed tomographic planning.
\end{abstract}

\section{KEYWORDS}

aortic valve stenosis, cardiac surgery, minimally invasive, minimally invasive cardiac surgery, minimally invasive surgical aortic valve replacement, RALT, right anterior minithoracotomy, SAVR, surgical aortic valve replacement

\section{1 | INTRODUCTION}

Minimally invasive aortic valve replacement (MIAVR) was first described by Cosgrove and Sabik ${ }^{1}$ in 1996. MIAVR is increasingly accepted in the surgical community with outcomes equivalent or superior to those of conventional $\mathrm{AVR}^{2}$ In addition to the smaller incision and improved cosmetic result, numerous studies report a reduction in postoperative bleeding, transfusion requirements, rates of atrial fibrillation, length of mechanical ventilation, length of intensive care unit and hospital stay, as well as postoperative pain with no difference in mortality. ${ }^{3-5}$ Furthermore, right anterolateral minithoracotomy (RALT), with complete preservation of the sternum, has been proposed as an even less-invasive approach for MIAVR. ${ }^{6}$ Surgical aortic valve replacement (SAVR) has been shown to improve symptoms and survival, yet older patients are at an increased risk of morbidity and mortality, making a less-invasive treatment strategy desirable in such patients. ${ }^{7}$ Studies have shown that up to $40 \%$ of patients over the age of 70 are denied aortic valve surgery based on age and higher risk profiles. ${ }^{8,9}$ However, surgical MIAVR has been shown to reduce morbidity and decrease mortality in high-risk

This is an open access article under the terms of the Creative Commons Attribution-NonCommercial-NoDerivs License, which permits use and distribution in any medium, provided the original work is properly cited, the use is non-commercial and no modifications or adaptations are made.

(c) 2020 The Authors. Journal of Cardiac Surgery published by Wiley Periodicals LLC 
populations. ${ }^{7,10-12}$ Here, we describe our approach to MIAVR by way of RALT. We also aim to define and classify the preoperative anatomical ascending aorta-sternal relationship as it is decisive in preoperative planning. We provide information about additional technical features and procedures that we have found to facilitate this challenging surgical procedure.

\section{MATERIALS AND METHODS}

\section{1 | Patient selection and preoperative planning}

Patients who are eligible for SAVR, according to the 2017 ESC/ EACTS guidelines for the management of valvular heart disease, ${ }^{13}$ need to be thoroughly evaluated regarding their suitability for the RALT approach. The reported exclusion criteria for SAVR-RALT are aneurysmal dilatation of the ascending aorta, ascending aorta located completely retrosternal or relatively left lateral, pathological calcification of the ascending aorta (soft plaques) or prior cardiac surgery, history of right-sided pleuritis, or a deep chest. ${ }^{14-16}$ In a preoperative computed tomography (CT) scan with contrast enhancement, we evaluate the anatomical relationship between intercostal spaces (ICS), sternum, ascending aorta and aortic valve (Figure 1). Regarding anatomical suitability, we find the distance between the planned
RALT incision and the aortic annular plane to be of great importance. Using three-dimensional (3D) CT reconstructions of the patient's thoracic cage, we can predict precisely which ICS we should enter to achieve optimal exposure. We propose a minithoracotomy to an aortic annular plane distance not exceeding $16 \mathrm{~cm}$. Additionally, we analyze the anatomic ascending aorta-sternal relationship as summarized in Figure 2. We consider patients with type la and type lb configurations suitable for SAVR-RALT. Aligning with previously published criteria, ${ }^{16}$ type II and type III ascending aorta-sternal configurations would be defined as unsuitable for SAVR-RALT. Nevertheless, we have been able to show an intraoperative rightward shift of the ascending aorta by application of single left lung (SLL) positive-endexpiratory-pressure (PEEP) ventilation of $20 \mathrm{~mm} \mathrm{Hg}$, thereby improving exposure and facilitating aortic cross-clamping (see below). Accordingly, patients with a preoperative type II configuration, hence classified as unsuitable, can be converted to a type lb configuration and thus become suitable for SAVR-RALT (Figure 3).

With transcatheter aortic valve implantation procedures becoming more popular, it is important to offer alternative surgical operations that come with both, known durability and suitability for a potential future transcatheter valve-in-valve option and a minimized surgical trauma with satisfactory cosmesis. As demonstrated in this manuscript, the SAVR-RALT procedure seems to complement both of these requirements.
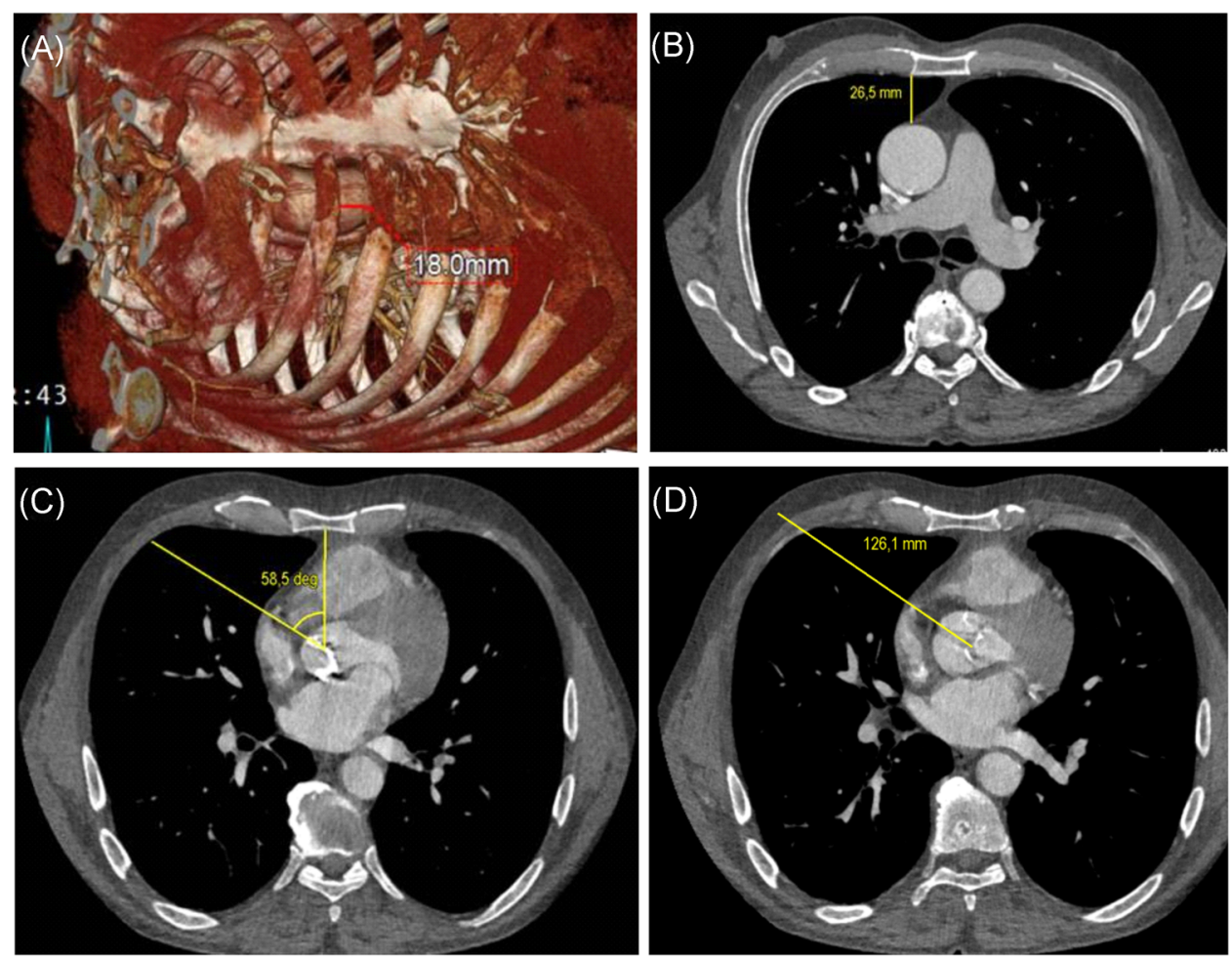

FIGURE 1 Preoperative imaging and procedural planning are crucial in MIAVR-RALT. A, Three-dimensional CT reconstruction (via Aquarius iNtuition Viewer [TeraRecon] software) for precise planning of the surgical strategy. B, Type lb preoperative ascending aorta-sternal relationship: distance from ascending aorta to sternum is $2.65 \mathrm{~cm}$. C, $\alpha$-Angle is $58.5^{\circ}$. D, Planned incision to aortic annular plane distance: $12.61 \mathrm{~cm}$. CT, computed tomography; MIAVR, minimally invasive aortic valve replacement; RALT, right anterolateral minithoracotomy 


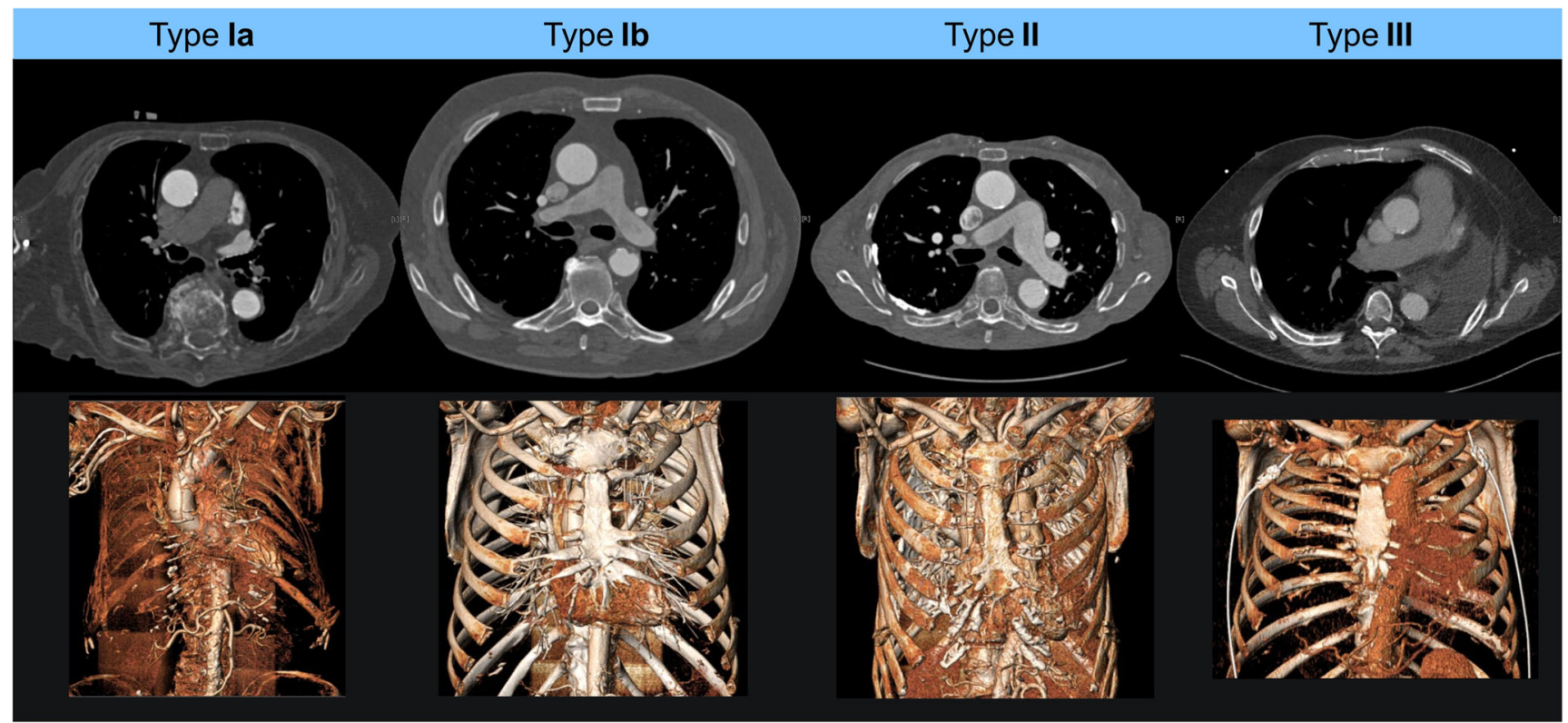

FIGURE 2 CT planning for RALT access: preoperative ascending aorta-sternal relationship. Four types of ascending aorta-sternal relationships at the level of the main pulmonary artery: Type la: ascending aorta is completely rightward from the sternum; Type Ib: ascending aorta more than $50 \%$ located on the right side of the sternum; Type II: >50\% of the ascending aorta underneath the sternum; Type III: ascending aorta more than $50 \%$ located on the left of the sternum. CT, computed tomography; RALT, right anterolateral minithoracotomy

Furthermore, patients with an aneurysmal dilatation or pathological calcification of the ascending aorta, or a history of prior cardiac surgery, right-sided pleuritis, partial pneumectomy, or a very deep chest should not be considered eligible for SAVR-RALT. Bicuspid stenotic aortic valves are treatable via this technique, however utmost attention should be paid to anatomic anomalies of the origin of the coronary ostia.

\section{2 | Operative technique}

\subsection{1 | Patient positioning and anesthetics}

The patient is intubated with a single-lumen or double-lumen endotracheal tube (in case of planned SLL-PEEP) and placed in a supine

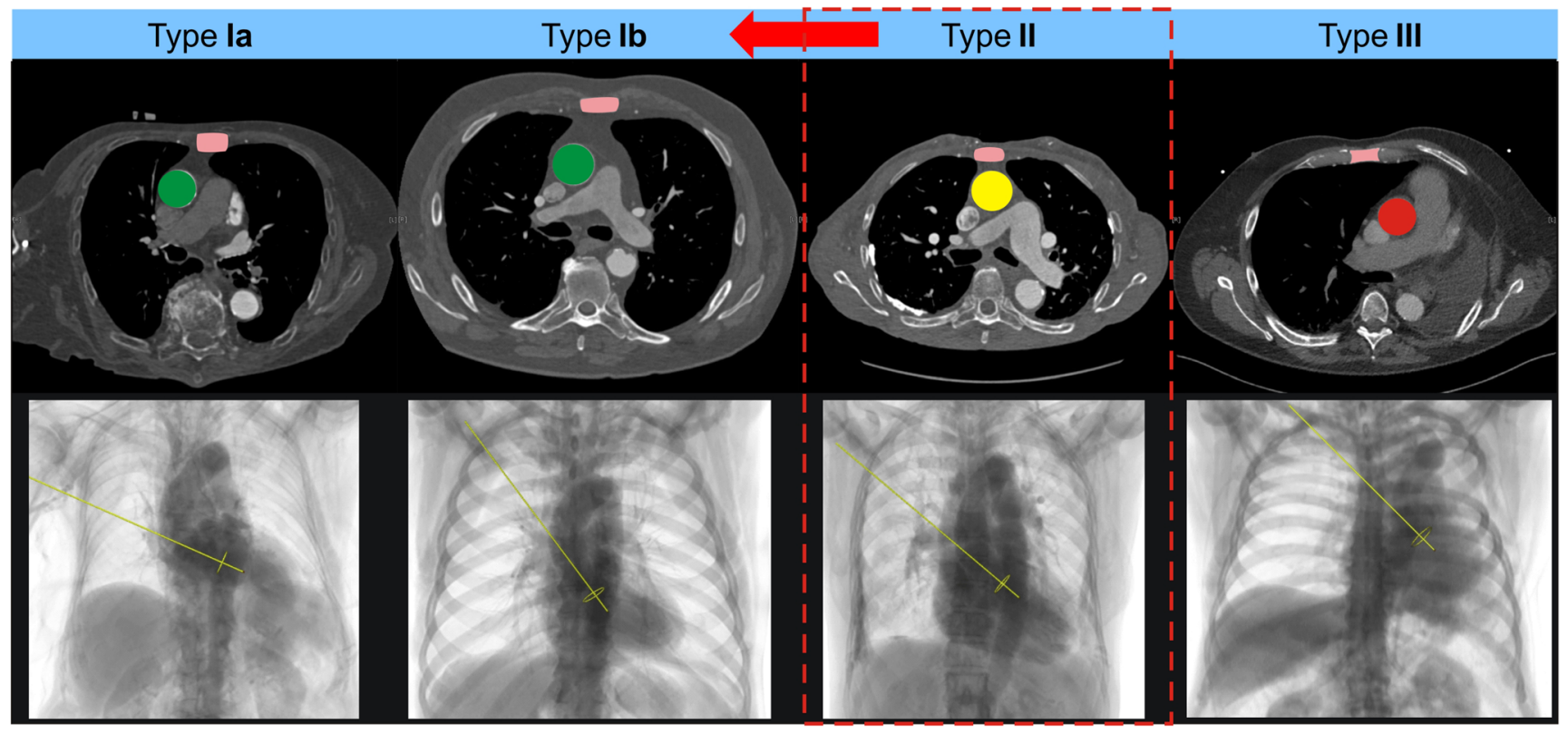

FIGURE 3 CT planning for RALT access: preoperative ascending aorta-sternal relationship. Theoretically, application of $20 \mathrm{~mm}$ Hg SLL-PEEP ventilation can shift the ascending aorta rightward to convert an anatomically unsuitable configuration into a suitable one. (red arrow). CT, computed tomography; RALT, right anterolateral minithoracotomy, SLL-PEEP, single left lung-positive-end-expiratory-pressure 
position after induction of general anesthesia. Defibrillator pads are placed on the chest wall. A transesophageal echocardiography (TEE) probe is placed and a thorough echocardiographic evaluation is performed.

\subsection{2 | Cannulation and cardiopulmonary bypass: percutaneous femoral cannulation}

Cardiopulmonary bypass (CPB) is established by percutaneous cannulation of the left common femoral artery and the right common femoral vein (single venous cannula; self-expanding smart cannula, LLC, Lausanne, Switzerland). The cannulas are placed in the superior vena cava and femoral artery under TEE guidance. For arterial cannulation, two suturemediated closure systems (Perclose ProGlide; Abbott Vascular, Clonmel, Tipperary, Ireland) are placed before insertion of the cannula. If 3D CT reconstructions of the femoral vessels indicate the right groin as more suitable for cannulation, the right femoral artery is selected as an arterial puncture site, as shown in Figure 4. In general, a 16-, or 18-FR arterial cannula is sufficient depending on patient size and arterial diameter.

If peripheral vascular disease is suspected at the time of femoral cannulation or grade 4 or 5 atherosclerotic disease is evident by TEE, axillary artery cannulation for antegrade arterial perfusion could be performed. Venous drainage is augmented with vacuum assistance $(-20$ to $-35 \mathrm{~mm} \mathrm{Hg}$ ) to decompress the right heart. After the establishment of CPB, a vent is inserted through the right superior pulmonary vein. Body temperature is maintained at around $34^{\circ} \mathrm{C}$. We use goal-directed-perfusion with $\mathrm{DO}_{2}$-guided perfusion, which is helpful in regard to optimal venous drainage as it allows you to safely reduce the pump-flow to $70 \%-80 \%$ of the calculated flow if required.

\subsection{3 | Thoracotomy and incisional}

A small 4- to 5-cm RALT is used to enter the thorax through the second or third ICS (third ICS most common). A soft-tissue retractor and an intercostal rib spreader provide additional exposure. We do not perform rib-transection/resection. The right internal mammary and vein are left untouched.

\subsection{4 | Intrathoracic exposure}

The pericardium is opened over the aorta and the incision extended down towards the inferior vena cava. Carefully planned placement of pericardial stay sutures is key to obtain adequate exposure of the aorta and valve. The sutures are brought out by a suture hook through a stab incision one or two ICS above the thoracotomy (Figure 5). If the ascending aorta is located directly under or on the left side of the sternum, a PEEP of $20 \mathrm{~mm} \mathrm{Hg}$ can be applied to the left lung only, inducing a rightward shift of the ascending aorta. This would be performed right before crossclamping to enhance aortic exposure and facilitate positioning of the aortic clamp.

\subsection{5 | Video-assisted endoscopic monitoring}

After entering the thorax, a high-definition standard $30^{\circ}$-thoracoscope is placed into the chest via a $5-\mathrm{mm}$ port through the second ICS at the right anterior axillary line. It not only provides an additional view from which to perform subsequent work but also brightly illuminates the entire chest. Throughout the procedure, the surgical field is flooded with $\mathrm{CO}_{2}(2 \mathrm{~L} / \mathrm{min})$ through this port.

\subsubsection{Aortic cross-clamping and cardioplegia}

The transthoracic external aortic cross-clamp is inserted via the third ICS at the right midaxillary line. Antegrade cardioplegia is delivered directly into the aortic root through a long needle or selectively into the coronary ostia and repeated, if necessary.
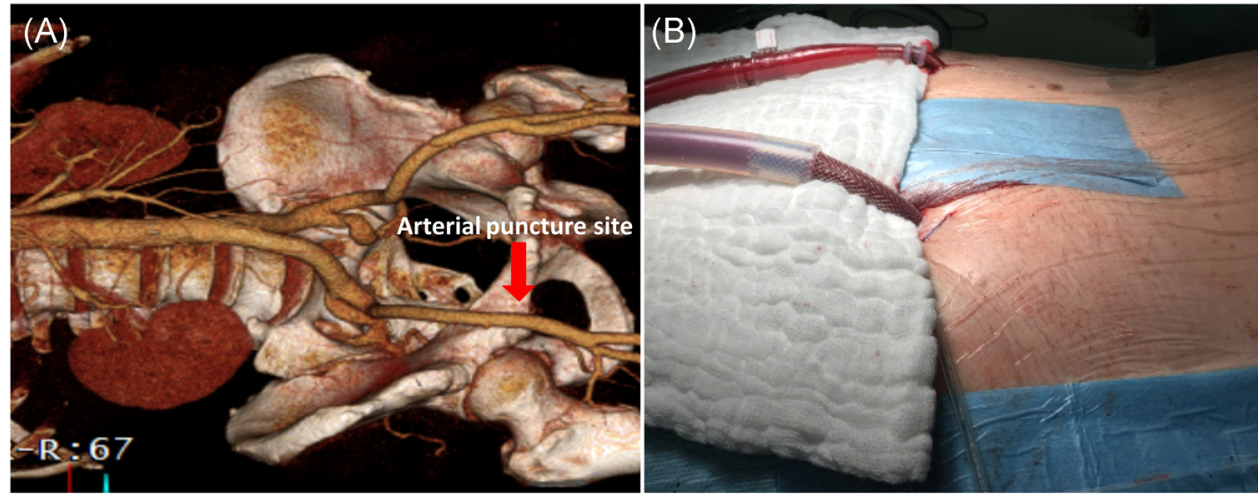

FIGURE 4 Femoral cannulation for CPB: preoperative planning of the femoral access site and percutaneous cannulation. A, Three-dimensional CT reconstruction of the femoral vessels: the femoral bifurcation is more cranial on the left than on the right side. The right common femoral artery, therefore, is the preferred arterial puncture platform for CPB. B, Percutaneous cannulation for CPB: right femoral artery for retrograde arterial perfusion and left femoral vein for venous drainage. CPB, cardiopulmonary bypass; $\mathrm{CT}$, computed tomography 

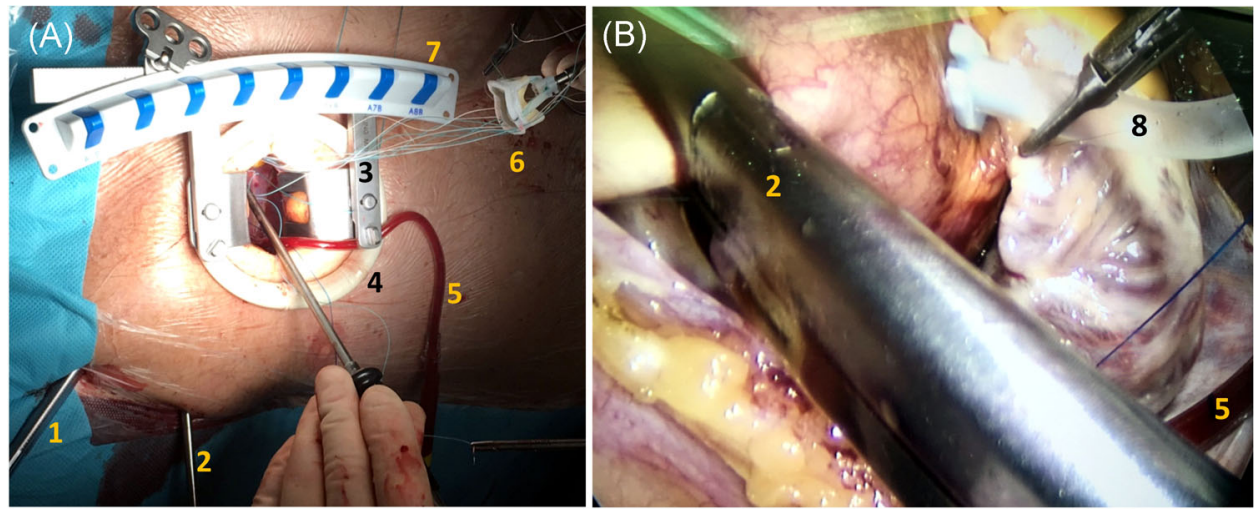

FIGURE 5 "The Berlin Setup" for minimally invasive surgical aortic valve replacement through a right anterior minithoracotomy. A, The surgeon's perspective: right anterior minithoracotomy: 1 = high-definition standard $30^{\circ}$-thoracoscope, $2=$ transthoracic external aortic cross-clamp, 3 = rib spreader, 4 = soft-tissue retractor, $5=$ left ventricular vent, $6=$ bioprosthetic aortic valve, and $7=$ suture organizer. $\mathrm{B}$, Intraoperative endoscopic screenshot from the high-definition standard $30^{\circ}$-thoracoscope: 2 = transthoracic cross-clamp, $5=$ left ventricular vent, and $8=$ cardioplegia line

\subsection{7 | Aortic valve exposure and aortic valve replacement}

The aortic valve is accessed through a hockey-stick aortotomy incision. To enhance exposure, both sides of the aortotomy are retracted anteriorly and laterally with stay sutures. Valve resection and annular debridement are carried out under direct vision. All procedures are performed with specially designed long-shafted instruments. Additionally, a standard aortic wall retractor is used by the assistant to optimize visualization of the annulus. Sizing and suture placement are performed in standard fashion. After delivering the prosthesis onto the annulus, all annular valve sutures are tied utilizing the MiniCor-Knot (LSI Solutions, Victor, NY) automated fastener device.

\subsection{8 | De-airing and closing}

Epicardial pacing wires are placed while the heart is still decompressed on CPB. Under TEE control, de-airing is performed antegradely via the aortotomy and retrogradely via vent-suction without direct manipulation of the heart. Separation from CPB, decannulation, TEE assessment of prosthesis function, and reversal of anticoagulation are all conducted as in conventional AVR. A standard chest tube is placed in the posterior pleural space. The pericardium is partially closed to limit adhesions of the lung to the heart for future interventions. A large pericostal suture is placed in a figure of eight fashion to approximate the ribs. The RALT incision is closed in routine fashion.

\section{3 | CONCLUSIONS}

There are several implications for surgical practice:

- The surgical learning curve and the greater complexity of the procedure should be taken into account in the planning. This, however, should not deter cardiac surgeons from providing advanced minimally invasive techniques to patients.

- The advantages of SAVR-RALT can be combined with accelerated mobilization, improved chest wall stability, and good cosmetic results.

- The RALT approach allows the sternum and ribs to be preserved in their entity. A sternum-sparing procedure decreases the potential of bleeding as well as the need for transfusions with the associated complications.

- Optimal patient preparation and correct surgical steps in the execution are significant factors for the success of this alternative access.

\section{ACKNOWLEDGMENTS}

The authors have no potential conflicts of interest relevant to this article. All SAVR-RALT procedures performed at the author's institution are in accordance with the ethical standards of the responsible local committee. Informed consent is always obtained from patients being treated with the RALT approach for SAVR. Additional informed consent was obtained from all individuals for whom identifying information is included in this article (eg, intraoperative pictures and preoperative CT scans).

\section{AUTHOR CONTRIBUTIONS}

All authors made contributions to the development of the described methods, contributed important intellectual content, and reviewed and approved the final version of the manuscript.

\section{REFERENCES}

1. Cosgrove DM 3rd, Sabik JF. Minimally invasive approach for aortic valve operations. Ann Thorac Surg. 1996;62:596-597.

2. Murzi M, Cerillo AG, Gilmanov D, et al. Exploring the learning curve for minimally invasive sutureless aortic valve replacement. J Thorac Cardiovasc Surg. 2017;152(6):1537-1546.

3. Neely RC, Boskovski MT, Gosev I, et al. Minimally invasive aortic valve replacement versus aortic valve replacement through full sternotomy: the Brigham and Women's Hospital experience. Ann Cardiothorac Surg. 2015;4(1):38-48. 
4. Ramlawi B, Bedeir K, Lamelas J. Aortic valve surgery: minimally invasive options. Methodist Debakey Cardiovasc J. 2016;12(1):27-32.

5. Stoliński J, Plicner D, Grudzień G, et al. A comparison of minimally invasive and standard aortic valve replacement. J Thorac Cardiovasc Surg. 2016;152(4):1030-1039.

6. Semsroth S, Matteucci Gothe R, Raith YR, et al. Comparison of two minimally invasive techniques and median sternotomy in aortic valve replacement. Ann Thorac Surg. 2017;104(3):877-883.

7. Gilmanov D, Farneti PA, Ferrarini M, et al. Full sternotomy versus right anterior minithoracotomy for isolated aortic valve replacement in octogenarians: a propensity-matched study. Interact Cardiovasc Thorac Surg. 2015;20(6):732-741.

8. Bouma BJ, van den Brink RB, Zwinderman K, et al. Which elderly patients with severe aortic stenosis benefit from surgical treatment? An aid to clinical decision making. J Heart Valve Dis. 2004;13:374-381.

9. Lamelas J. Minimally invasive aortic valve replacement: the "Miami Method". Ann Cardiothorac Surg. 2015;4(1):71-77.

10. Bowdish ME, Hui DS, Cleveland JD, et al. A comparison of aortic valve replacement via an anterior right minithoracotomy with standard sternotomy: a propensity score analysis of 492 patients. Eur J Cardiothorac Surg. 2016;49(March 2015):456-463. https://doi.org/ 10.1093/ejcts/ezv038

11. Lamelas J, Sarria A, Santana O, Pineda AM, Lamas GA. Outcomes of minimally invasive valve surgery versus median sternotomy in patients age 75 years or greater. Ann Thorac Surg. 2011;91:79-84.

12. Santana O, Reyna J, Grana R, Buendia M, Lamas GA, Lamelas J. Outcomes of minimally invasive valve surgery versus standard sternotomy in obese patients undergoing isolated valve surgery. Ann Thorac Surg. 2011;91: 406-410.

13. Baumgartner H, Falk V, Bax JJ, et al. ESC/EACTS Guidelines for the management of valvular heart disease. The Task Force for the Management of Valvular Heart Disease of the European Society of Cardiology (ESC) and the European Association for Cardio-Thoracic Surgery (EACTS). Eur Heart J. 2017;38(36):2739-2791.

14. Fattouch K, Moscarelli M, Del Giglio M, et al. Non-sutureless minimally invasive aortic valve replacement: mini-sternotomy versus minithoracotomy: a series of 1130 patients. Interact Cardiovasc Thorac Surg. 2016;23(2):253-258.

15. Andreas M, Mahr S, Kocher A, Laufer G. Minimalinvasiver Aortenklappenersatz über eine anteriore rechtsseitige Thorakotomie. Zeitschrift für Herz-, Thorax- und Gefäßchirurgie. 2017;31(4):241-246.

16. Glauber M, Ferrarini M, Miceli A. Minimally invasive aortic valve surgery: state of the art and future directions. Ann Cardiothorac Surg. 2015;4(1):26-32.

How to cite this article: Van Praet KM, van Kampen A, Kofler $M$, et al. Minimally invasive surgical aortic valve replacement: The RALT approach. J Card Surg. 2020;35:2341-2346. https://doi.org/10.1111/jocs.14756 